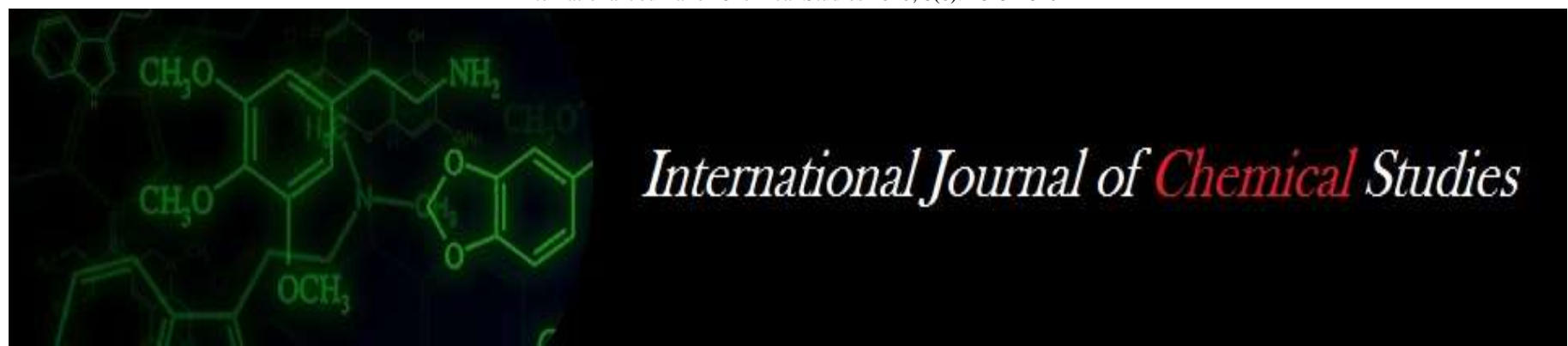

P-ISSN: 2349-8528

E-ISSN: 2321-4902

www.chemijournal.com

IJCS 2020; 8(6): 2815-2820

(C) 2020 IJCS

Received: 15-09-2020

Accepted: 21-10-2020

\section{Shivani Sharma}

Ph.D Scholar, Department of Silviculture and Agroforestry, Dr. YS Parmar University of Horticulture and Forestry, Nauni, Solan, Himachal

Pradesh, India

\section{KS Pant}

Professor, Department of Silviculture and Agroforestry, Dr. YS Parmar University of Horticulture and Forestry, Nauni, Solan, Himachal Pradesh, India

\section{Aruna Mehta}

Scientist, College of Horticulture and Forestry, Thunag, Mandi,

Himachal Pradesh, India
Corresponding Author: Shivani Sharma

Ph.D Scholar, Department of Silviculture and Agroforestry, Dr. YS Parmar University of Horticulture and Forestry,

Nauni, Solan, Himachal

Pradesh, India

\section{Effect of organic manures on soil physico- chemical properties of Ocimum sanctum under stone fruit based agroforestry system}

\author{
Shivani Sharma, KS Pant and Aruna Mehta
}

DOI: https://doi.org/10.22271/chemi.2020.v8.i6an.11208

\title{
Abstract
}

The study was conducted for two consecutive years during 2016 and 2017 at experimental farm of Department of Silviculture and Agroforestry, Dr YS Parmar University of Horticulture and Forestry, Nauni, Solan (HP) to evaluate the effect of different organic manures and their distance of application from tree trunk on soil physico-chemical properties under Peach and Apricot based agroforestry system. The experiment was laid out in randomized block design (factorial) consisting of seven treatments with three distance levels. The results revealed that among different organic manure treatments, FYM @ $25 \mathrm{t}$ $\mathrm{ha}^{-1}$ significantly influenced the soil physico-chemical properties of Ocimum sanctum and recorded highest soil moisture (11.20\% and $11.27 \%)$, electrical conductivity $(0.29$ and 0.29$)$, organic carbon (2.35\% and $2.12 \%$ ), available nitrogen (354.97 and 255.42), available phosphorus (55.08 and 53.88) and available potassium (356.40 and 278.96) over control when applied at a distance of $1 \mathrm{~m}$ away from tree trunk under peach and apricot based agroforestry system, respectively.

Keywords: Jeevamrut, Ocimum sanctum, peach, soil physico-chemical properties

\section{Introduction}

Soil health is a continuous potential of soil to function as a living system, which sustain biological productivity, environmental quality as well as plant \& animal health (Karlen et al., 1997; Oliver et al,. 2013) ${ }^{[1,2]}$. Sustainable agriculture production depends on the scientific management of soil health to meet people's present and future needs. But now days, the health of soil facing high degradation problems due to increased pressure of human and livestock population on inadequate natural resources. Unscientific agricultural practices, deforestation, over grazing and construction of roads are some of major causes responsible for degradation of soil health (Semwal et al., 2009; Araujo et al., 2012) ${ }^{[3,4]}$. Along with rising human population, climate change and unsustainable use of natural resources affects soil nutrient status of the country (Sarvade et al., 2014) ${ }^{[5]}$ which is an important factor for controlling yields of the crops. In future, sustainable soil conservation efforts would be needed for tackling problems such as soil health depletion, climate change and food insecurity.

Agroforestry is sustainable land use system and it has potential for improving soil physical, chemical and biological properties through multifunctional approach. The combination of trees, crops and livestock mitigates environmental risk, creates a permanent soil cover against soil erosion, minimizes damage from floods and acts as water storage thereby benefit crops and pastures (FAO, 2015) ${ }^{[6]}$. Nair (1984) ${ }^{[7]}$ has reported that agri-horticultural and agripastoral systems have the potential to reduce soil erosion, runoff and to maintain soil organic matter, improve soil physical properties and augment nitrogen fixation and promote efficient nutrient cycling. Agroforestry is also a viable option for climate change mitigation, sustainable development and has the potential to improve the socio-economic conditions of the farmers (Dutt and Thakur, 2004) ${ }^{[8]}$. Most of the Indian agricultural lands are deprived of some of the essential nutrients for growth and development of crop plants. Chemical fertilizers supplement the nutrient supply but such chemical fertilizers pose serious health hazards and microbial population problem in soil besides being quite expensive and making the cost of production high. The long term use of chemical fertilizers without organic supplements damages the soil properties and causes environmental pollution (Albiach et al., 2000) ${ }^{[9]}$. 
This scenario has encouraged scientists to use organic materials for improving soil properties and high crop production.

Ocimum sanctum is an aromatic plant distributed throughout the tropical and subtropical climates of Indian subcontinent up to an elevation of $900 \mathrm{~m}$ (Raina et al., 2013) ${ }^{[10]}$. The crop is highly profitable due to the presence of a complex mixture of volatile substances, monoterpenes, sesquiterpenes and their oxygenated analogs present at low concentrations in plants (Lucchesi et al., 2004) [11]. Essential oil of Ocimum sanctum finds extensive role in pharmaceutical preparations, perfumes, cosmetics and also as flavoring agent in food items (Raina et $a l ., 2013)^{[10]}$. The species is traditionally used in the health care as expectorant, analgesic, anticancer, antidiabetic, antistress, antioxidant and wound healing. Though the production of the species can be increased by supplying the nutrients through chemical fertilizers alone but continuous use of the same on long-term basis may lead to the degradation of the soil health. Usage of organic manures generally improves the soil physical, chemical, biological properties and moisture holding capacity thus helps in enhanced crop productivity. Keeping in view the above facts, the present study was conducted in order to assess the effect of different organic manure doses on soil physico-chemical properties of Ocimum sanctum under stone fruit based agroforestry system.

\section{Materials and Methods}

The present investigation was conducted at the experimental farm of Department of Silviculture and Agroforestry, Dr. Y S Parmar University of Horticulture and Forestry, Nauni, Solan (H.P.). The experiment comprised of seven treatments viz., $\mathrm{T}_{1}$ (15t/ha FYM), T T $_{2}$ (20t/ha FYM), $\mathrm{T}_{3}(25 \mathrm{t} / \mathrm{ha} \quad \mathrm{FYM}), \mathrm{T}_{4}$ (180ml/plant Jeevamrut), $\mathrm{T}_{5}(300 \mathrm{ml} /$ plant Jeevamrut $), \mathrm{T}_{6}$
(420 ml/plant Jeevamrut) and $\mathrm{T}_{7}$ (no manure-control) and three distance levels of application from tree trunk viz., $1 \mathrm{~m}$, $2 \mathrm{~m}$ and $3 \mathrm{~m}$ away from tree. The experiment was laid out in randomized block design with three replications. This study consisted of fruit trees viz; Peach var. nectarine (Prunus persica) and Apricot (Prunus armeniaca) as woody perennial and Ocimum sanctum as intercrop in agrisilviculture system. Fruit trees were planted in East to West direction at a distance of $9 \mathrm{~m} \times 4 \mathrm{~m}$. While, the plot size of $3 \mathrm{~m} \times 3 \mathrm{~m}$ and spacing of $45 \mathrm{~cm} \times 45 \mathrm{~cm}$ was maintained for Ocimum sanctum. Seedlings of Ocimum sanctum were transplanted in the month of June. Light irrigations were given after transplanting to facilitate the establishment of seedlings. The experimental plots were maintained properly and kept free from weeds. FYM was evenly spread and mixed with the soil before transplanting. Thereafter, Jeevamrut (5\%) was applied as soil drench @ 30, $50,70 \mathrm{ml}$ per plant after the 30 days of transplanting. Afterwards application was done at 15 days interval till the final harvesting is done (full bloom stage of flowering) with total 6 applications. Thus making total application of $180 \mathrm{ml}$ (Palekar, 2006) ${ }^{[12]}, 300 \mathrm{ml}$ and $420 \mathrm{ml}$ per plant (Basavaraj et al., 2016) ${ }^{[13]}$. Soil samples were collected from $0-15 \mathrm{~cm}$ depth at three distances from tree trunk ( $1 \mathrm{~m}, 2 \mathrm{~m}$ and $3 \mathrm{~m}$ away from tree trunk) from each plot before transplanting as well as at the time of harvesting of the crop and collected samples were air dried under shade in laboratory; crushed using wooden mortar and pestle and then sieved through $2 \mathrm{~mm}$ plastic sieve. The observations on physico-chemical properties of soil viz., soil moisture $(\%)$, soil $\mathrm{pH}$, electrical conductivity $\left(\mathrm{ds} / \mathrm{m}^{-1}\right)$, organic carbon $(\%)$, available nitrogen $(\mathrm{kg} / \mathrm{ha})$, available phosphorus ( $\mathrm{kg} / \mathrm{ha})$ and available potassium ( $\mathrm{kg} / \mathrm{ha})$ were recorded. The methods and instruments used for soil physical and chemical analysis have been given in Table 1 .

Table 1: Methods and instrument used for soil chemical analysis.

\begin{tabular}{|c|c|c|c|}
\hline Sr. No. & Parameters & Methods employed & Instrument/ apparatus used \\
\hline 1 & Soil moisture & Gravimetric method & 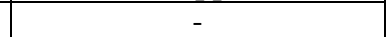 \\
\hline 2 & Electrical conductivity & 1:2 soil water suspension (Jackson, 1973) ${ }^{[14]}$ & Electrical conductivity meter \\
\hline 3 & $\mathrm{pH}$ & 1:2 Soil water suspension (Jackson, 1973) ${ }^{[14]}$ & $\mathrm{pH}$ meter \\
\hline 4 & Organic carbon & Rapid titration method (Walkey and Black, 1934) ${ }^{[15]}$ & - \\
\hline 5 & Soil nitrogen & Alkaline potassium permangante method (Subbiah and Asija, 1956) ${ }^{[16]}$ & Kjeldhal distillation unit \\
\hline 6 & Phosphorous $\left(\mathrm{P}_{2} \mathrm{O}_{5}\right)$ & Olsen et al. $(1954)^{[17]}$ & Spectronic 20-D+ \\
\hline 7 & Potassium & Neutral $1 \mathrm{~N}$ ammonium acetate solution method (Merwin and Peech 1951) ${ }^{[18]}$ & Flame Photometer \\
\hline
\end{tabular}

The entire data of the present study were statistically analyzed by using analysis of variance (ANOVA) for Randomized Block Design (RBD) in accordance with the procedure outlined by Gomez and Gomez (1984) [19] where effects exhibited significance at 5 per cent level of probability and then critical difference (CD) was calculated.

\section{Results and Discussion \\ Soil moisture (\%)}

Data presented in Table 2 showed that with increase in distance from the tree, soil moisture was significantly decreased. The maximum soil moisture of $9.90 \%$ and $9.89 \%$ was recorded at a distance of $1 \mathrm{~m}$ away from tree trunk $\left(\mathrm{D}_{1}\right)$ under peach and apricot tree, respectively. However, the minimum soil moisture $(9.19 \%$ and $9.14 \%)$ was observed in $\mathrm{D}_{3}(3 \mathrm{~m}$ away from tree trunk) under peach and apricot tree, respectively. Tripathi (2012) ${ }^{[20]}$ has reported that moisture content, NPK and other macronutrient were higher under tree cover than in open condition. Among various organic manures treatment, the maximum soil moisture $(10.93 \%$ and $10.77 \%)$ was recorded in $\mathrm{T}_{3}\left(25 \mathrm{FYM} \mathrm{ha}^{-1}\right)$ under peach and apricot tree, respectively. Similarly, Acharya et al. (1988) [21] have also reported beneficial effects on soil structural index, infiltration rate and water retention characteristics of soil with addition of FYM. However, the minimum soil moisture (8.29 $\%$ and $8.17 \%$ ) was observed in $\mathrm{T}_{7}$ (control-no manure) under peach and apricot tree, respectively. The interaction between different organic manures and canopy exerted a nonsignificant effect on soil moisture. However, maximum soil moisture was recorded in $\mathrm{T}_{3} \mathrm{D}_{1}(11.20 \%$ and $11.27 \%)$ and minimum in $\mathrm{T}_{4} \mathrm{D}_{3}(8.38 \%$ and $8.24 \%)$ under peach and apricot tree, respectively.

\section{$\mathrm{EC}\left(\mathrm{dSm}^{-1}\right)$ and $\mathrm{pH}$}

The data presented in tables 3 and 4 revealed that organic manure and their distance of application exerted a significant effect on electrical conductivity and $\mathrm{pH}$. The maximum electrical conductivity (0.24 and 0.23$)$ and $\mathrm{pH}$ (7.12 and 7.30) was recorded at a distance of $1 \mathrm{~m}$ away from tree trunk $\left(\mathrm{D}_{1}\right)$ under peach and apricot tree, respectively. However, the minimum electrical conductivity $(0.21$ and 0.20$)$ and $\mathrm{pH}(6.54$ and 6.64) was observed in $\mathrm{D}_{3}$ ( $3 \mathrm{~m}$ away from tree trunk) 
under peach and apricot tree, respectively. Yogeshwari (2015) [22] has also reported a significant decline in all soil parameters except bulk density, soil $\mathrm{pH}$ and soil iron content with increase in distance from the tree. Among various organic manure treatments, the maximum electrical conductivity (0.28 and 0.27$)$ and $\mathrm{pH}(7.06$ and 7.15$)$ was recorded in $\mathrm{T}_{3}\left(25 \mathrm{t} \mathrm{FYM} \mathrm{ha}^{-1}\right)$ under peach and apricot tree, respectively. However, the minimum electrical conductivity (0.19 and 0.19) and $\mathrm{pH}\left(6.37\right.$ and 6.36) was observed in $\mathrm{T}_{7}$ (control) under peach and apricot tree, respectively. The interaction between different organic manures and canopy exerted a non-significant effect on $\mathrm{EC}$ and $\mathrm{pH}$. However, maximum electrical conductivity $(0.29$ and 0.29$)$ was recorded in $\mathrm{T}_{3} \mathrm{D}_{2}$ and maximum $\mathrm{pH}$ (7.52 and 7.62) was recorded in $\mathrm{T}_{2} \mathrm{D}_{1}$. Bowen et al. (1988) ${ }^{[23]}$ have also reported that plant residues or litter has multi-beneficial effects on maintenance of soil physical conditions, soil organic matter, provision of nutrients and stimulation of biological activity as well as moderately acidity in soil.

\section{Organic carbon NP and K}

It is evident from the tables 5, 6, 7 and 8 that different organic manures and their distance of application exerted a significant effect on organic carbon, $\mathrm{N}, \mathrm{P}$ and $\mathrm{K}$. Maximum organic carbon $(1.94 \%$ and $1.75 \%)$, available nitrogen $(325.18 \mathrm{~kg} / \mathrm{h}$ and $316.83 \mathrm{~kg} / \mathrm{h})$, available phosphorus $(50.16 \mathrm{~kg} / \mathrm{h}$ and 49.41 $\mathrm{kg} / \mathrm{h})$ and available potassium $(291.15 \mathrm{~kg} / \mathrm{h}$ and $247.67 \mathrm{~kg} / \mathrm{h})$ were recorded at $\mathrm{D}_{1}$ (distance of $1 \mathrm{~m}$ away from tree trunk) under peach and apricot tree, respectively. In the present study, the reason for higher organic carbon could be more leaf litter addition at $\mathrm{D}_{1}$ distance as compared to $\mathrm{D}_{3}$ distance. Our findings can be supported with results of Khybri et al. (1992) [24] who reported that planting trees on field boundaries increased organic carbon, available nitrogen, available phosphorus and potash. Whereas, the minimum organic carbon $(1.45 \%$ and $1.35 \%)$, available nitrogen $(306.27 \mathrm{~kg} / \mathrm{h}$ and $306.17 \mathrm{~kg} / \mathrm{h}$ ), available phosphorus $(40.92 \mathrm{~kg} / \mathrm{h})$ and available potassium $(265.43 \mathrm{~kg} / \mathrm{h}$ and $235.11 \mathrm{~kg} / \mathrm{h})$ was observed in $\mathrm{D}_{3}$ ( $3 \mathrm{~m}$ away from tree trunk) under peach and apricot tree, respectively except for available phosphorous under apricot tree which was recorded minimum in $\mathrm{D}_{2}$ (39.82). Similarly, Bhat (2015) ${ }^{[25]}$ have also reported maximum available $\mathrm{N}, \mathrm{P}$, and $\mathrm{K}$ content in soil in $\mathrm{S}_{2}$ treatment (near the tree i.e. $8 \times 4 \mathrm{~m}$ ) under intercropped conditions as compared to $\mathrm{S}_{0}$ (sole crop).

Among various organic manure treatments, the maximum organic carbon $(2.26 \%$ and $2.04 \%)$, available nitrogen $(346.18 \mathrm{~kg} / \mathrm{h}$ and $258.36 \mathrm{~kg} / \mathrm{h})$, available phosphorus $(49.17$ $\mathrm{kg} / \mathrm{h}$ and $48.09 \mathrm{~kg} / \mathrm{h})$ and available potassium $(331.03 \mathrm{~kg} / \mathrm{h}$ and $265.41 \mathrm{~kg} / \mathrm{h})$ was recorded in $\mathrm{T}_{3}\left(25 \mathrm{t} \mathrm{FYM} \mathrm{ha}{ }^{-1}\right)$ under peach and apricot tree, respectively. Bellakki and Badanur (1997) ${ }^{[26]}$ have reported increased organic carbon content with incorporation of FYM or sunnhemp to soil. Similarly, another author (Balaji, 1994) ${ }^{[27]}$ has also noticed higher levels of total nitrogen with application of organic manure either vermicompost or FYM in combination with chemical fertilizers over control (no manure). However, the minimum organic carbon $(1.27 \%$ and $1.14 \%)$, available nitrogen $(347.10 \mathrm{~kg} / \mathrm{h}$ and $265.51 \mathrm{~kg} / \mathrm{h})$, available phosphorus $(33.07$ $\mathrm{kg} / \mathrm{h}$ and $31.50 \mathrm{~kg} / \mathrm{h})$ and available potassium $(229.20 \mathrm{~kg} / \mathrm{h}$ and $224.07 \mathrm{~kg} / \mathrm{h}$ ) was observed in $\mathrm{T}_{7}$ (control) under peach and apricot tree, respectively. Considering the Jeevamrut doses, soil organic carbon (1.93\%) was found maximum in $\mathrm{T}_{6}$ (420ml/plant Jeevamrut). Chandrakala (2008) ${ }^{[28]}$ has reported that application of liquid manures also lowered the bulk density and increased the organic carbon content. The interaction between different organic manures and canopy exerted a non-significant effect on all soil physic-chemical parameters, except for available phosphorus under Apricot tree where interaction between treatments and different distances from tree exerted significant effect. However, maximum available phosphorous $(53.88 \mathrm{~kg} / \mathrm{h})$ was recorded at a distance of $1 \mathrm{~m}$ away from tree when FYM was applied @ $25 \mathrm{t} \mathrm{ha}^{-1}\left(\mathrm{~T}_{3}\right)$ which was statistically at par with $\mathrm{T}_{6} \mathrm{D}_{1}$. Available Phosphorus was also reported higher where plants were grown under tree and supplied with $25 \mathrm{tha}^{-1}$ FYM (Gulabrao, 2016) ${ }^{[29]}$. Highest available K content of soil has also been reported with the application of FYM by Sharma and Sharma (2002) ${ }^{[30]}$.

\section{Conclusion}

The application of organic manures had a significant effect on soil physico-chemical properties of Ocimum sanctum with superior performance at FYM @ $25 \mathrm{t} \mathrm{ha}^{-1}$. All the soil physico-chemical parameters viz., soil moisture $(11.20 \%$ and $11.27 \%)$, electrical conductivity $\left(0.29 \mathrm{dSm}^{-1}\right.$ and $\left.0.29 \mathrm{dSm}^{-1}\right)$, organic carbon $(2.35 \%$ and $2.12 \%)$, available nitrogen $(354.97 \mathrm{~kg} / \mathrm{h}$ and $255.42 \mathrm{~kg} / \mathrm{h})$, available phosphorus $(55.08$ $\mathrm{kg} / \mathrm{h}$ and $53.88 \mathrm{~kg} / \mathrm{h})$ and available potassium $(356.40 \mathrm{~kg} / \mathrm{h}$ and $278.96 \mathrm{~kg} / \mathrm{h}$ ) were highest when a dose of FYM @ $25 \mathrm{t}$ $\mathrm{ha}^{-1}$ was applied at a distance of $1 \mathrm{~m}$ away from tree trunk under peach and apricot based agroforestry system, respectively. This study emphasizes the potential use of organic manures in fruit-based agroforestry systems as an efficient strategy to maximize land use efficiency and improve soil health.

Table 2: Effect of organic manure and distance from tree trunk on soil moisture (\%) under different agroforestry system.

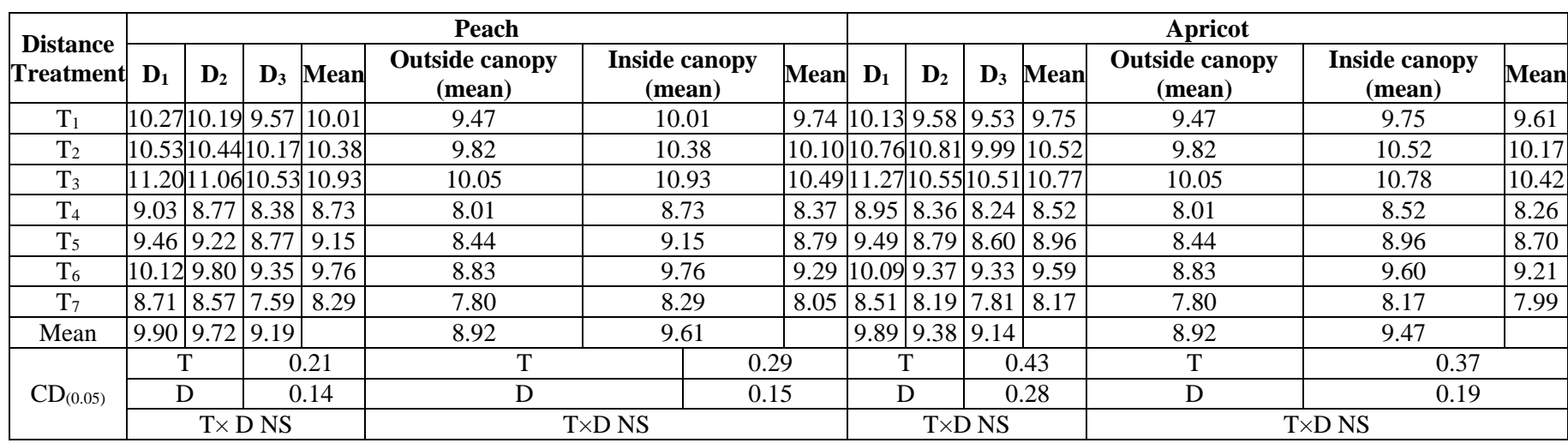

* $\mathrm{T}=$ Tree; $\mathrm{D}=$ distance of application from tree trunk; $\mathrm{TxD}=$ interaction between tree and distance of application from tree trunk. 
Table 3: Effect of organic manure and distance from tree trunk on EC $\left(\mathrm{dSm}^{-1}\right)$ under different agroforestry system.

\begin{tabular}{|c|c|c|c|c|c|c|c|c|c|c|c|c|c|c|}
\hline \multirow{2}{*}{$\begin{array}{c}\text { Distance } \\
\text { Treatme } \\
\text { nt }\end{array}$} & \multicolumn{7}{|c|}{ Peach } & \multicolumn{7}{|c|}{ Apricot } \\
\hline & $\mathbf{D}_{1}$ & $\mathbf{D}_{2}$ & $\mathbf{D}_{3}$ & Mean & $\begin{array}{c}\text { Outside canopy } \\
\text { (mean) }\end{array}$ & $\begin{array}{c}\text { Inside canopy } \\
\text { (mean) }\end{array}$ & Mean & $\mathbf{D}_{1}$ & $\mathbf{D}_{2}$ & $\mathbf{D}_{3}$ & Mean & $\begin{array}{c}\text { Outside canopy } \\
\text { (mean) }\end{array}$ & $\begin{array}{c}\text { Inside canopy } \\
\text { (mean) }\end{array}$ & Mean \\
\hline $\mathrm{T}_{1}$ & 0.25 & 0.24 & 0.21 & 0.23 & 0.19 & 0.23 & 0.21 & 0.23 & 0.23 & 0.20 & 0.22 & 0.19 & 0.22 & 0.21 \\
\hline $\mathrm{T}_{2}$ & 0.26 & 0.26 & 0.23 & 0.25 & 0.19 & 0.25 & 0.22 & 0.25 & 0.25 & 0.21 & 0.24 & 0.19 & 0.24 & 0.21 \\
\hline $\mathrm{T}_{3}$ & 0.29 & 0.29 & 0.27 & 0.28 & 0.20 & 0.28 & 0.24 & 0.29 & 0.28 & 0.25 & 0.27 & 0.20 & 0.27 & 0.24 \\
\hline $\mathrm{T}_{4}$ & 0.24 & 0.21 & 0.19 & 0.21 & 0.18 & 0.21 & 0.20 & 0.23 & 0.20 & 0.19 & 0.21 & 0.18 & 0.21 & 0.19 \\
\hline $\mathrm{T}_{5}$ & 0.22 & 0.21 & 0.19 & 0.20 & 0.17 & 0.20 & 0.19 & 0.21 & 0.20 & 0.18 & 0.20 & 0.17 & 0.20 & 0.18 \\
\hline $\mathrm{T}_{6}$ & 0.21 & 0.21 & \begin{tabular}{|l|}
0.18 \\
\end{tabular} & 0.20 & 0.16 & 0.20 & 0.18 & 0.21 & 0.20 & 0.18 & 0.20 & 0.16 & 0.20 & 0.18 \\
\hline $\mathrm{T}_{7}$ & 0.20 & 0.19 & 0.18 & 0.19 & 0.17 & 0.19 & 0.18 & 0.19 & 0.18 & 0.17 & 0.19 & 0.17 & 0.19 & 0.18 \\
\hline Mean & 0.24 & 0.23 & 0.21 & & 0.18 & 0.23 & & 0.23 & 0.22 & 0.20 & & 0.18 & 0.22 & \\
\hline \multirow{3}{*}{$\mathrm{CD}_{(0.05)}$} & \multicolumn{2}{|c|}{$\mathrm{T}$} & & 0.01 & $\mathrm{~T}$ & \multicolumn{2}{|l|}{0.01} & \multicolumn{2}{|c|}{$\mathrm{T}$} & \multicolumn{2}{|r|}{0.01} & $\mathrm{~T}$ & \multicolumn{2}{|l|}{0.01} \\
\hline & \multicolumn{2}{|c|}{$\mathrm{D}$} & & 0.01 & $\mathrm{D}$ & \multicolumn{2}{|l|}{0.01} & \multirow{2}{*}{\multicolumn{2}{|c|}{$\mathrm{D}$}} & \multicolumn{2}{|r|}{0.01} & $\mathrm{D}$ & \multicolumn{2}{|l|}{0.01} \\
\hline & \multicolumn{2}{|c|}{$\mathrm{T} \times \mathrm{D}$} & & NS & $\mathrm{T} \times \mathrm{D}$ & \multicolumn{2}{|l|}{ NS } & $\mathrm{T} \times \mathrm{D}$ & & \multicolumn{2}{|r|}{ NS } & $\mathrm{T} \times \mathrm{D}$ & \multicolumn{2}{|l|}{ NS } \\
\hline
\end{tabular}

*T= Tree; $\mathrm{D}=$ distance of application from tree trunk; $\mathrm{T} x \mathrm{D}=$ interaction between tree and distance of application from tree trunk.

Table 4: Effect of organic manure and distance from tree trunk on soil $\mathrm{pH}$ under different agroforestry system.

\begin{tabular}{|c|c|c|c|c|c|c|c|c|c|c|c|c|}
\hline \multirow{2}{*}{$\begin{array}{c}\text { Distance } \\
\text { Treatment }\end{array}$} & \multicolumn{6}{|c|}{ Peach } & \multicolumn{6}{|c|}{ Apricot } \\
\hline & $D_{1}$ & $\mathbf{D}_{2}$ & D3 Mean & $\begin{array}{c}\text { Outside canopy } \\
\text { (mean) }\end{array}$ & $\begin{array}{c}\text { Inside canopy } \\
\text { (mean) }\end{array}$ & Mean & $D_{1}$ & $\mathbf{D}_{2}$ & D3 Mean & $\begin{array}{c}\text { Outside canopy } \\
\text { (mean) }\end{array}$ & $\begin{array}{c}\text { Inside canopy } \\
\text { (mean) }\end{array}$ & Mean \\
\hline $\mathrm{T}_{1}$ & \multicolumn{3}{|c|}{\begin{tabular}{|l|l|l|}
6.896 .63 & 6.47 & 6.66 \\
\end{tabular}} & 6.55 & 6.66 & 6.61 & \multicolumn{3}{|c|}{\begin{tabular}{|l|l|l|}
7.226 .79 & 6.54 & 6.85 \\
\end{tabular}} & 6.55 & 6.85 & 6.70 \\
\hline $\mathrm{T}_{2}$ & \multicolumn{3}{|c|}{\begin{tabular}{|l|l|l|}
7.526 .80 & 6.48 & 6.94 \\
\end{tabular}} & 6.22 & 6.94 & 6.58 & \multicolumn{3}{|c|}{\begin{tabular}{|c|c|c|}
7.626 .94 & 6.58 & 7.05 \\
\end{tabular}} & 6.22 & 7.05 & 6.64 \\
\hline $\mathrm{T}_{3}$ & \multicolumn{3}{|c|}{\begin{tabular}{|l|l|l|}
7.347 .09 & 6.74 & 7.06 \\
\end{tabular}} & 6.68 & 7.06 & 6.87 & \multicolumn{3}{|c|}{\begin{tabular}{|l|l|l|}
7.517 .11 & 6.84 & 7.15 \\
\end{tabular}} & 6.68 & 7.15 & 6.92 \\
\hline $\mathrm{T}_{4}$ & \multicolumn{3}{|c|}{\begin{tabular}{|l|l|l|}
7.187 .05 & 6.37 & 6.86 \\
\end{tabular}} & 6.41 & 6.86 & 6.64 & \multicolumn{2}{|c|}{\begin{tabular}{|l|l|l|}
7.317 .15 & 6.5 \\
\end{tabular}} & \begin{tabular}{|l|l|}
6.53 & 7.00 \\
\end{tabular} & 6.41 & 7.00 & 6.71 \\
\hline$T_{5}$ & \multicolumn{3}{|c|}{\begin{tabular}{|l|l|l|}
7.066 .547 .04 & 6.88 \\
\end{tabular}} & 6.63 & 6.88 & 6.76 & \multicolumn{2}{|c|}{7.406 .747 .17} & \begin{tabular}{|l|l|}
7.17 & 7.10 \\
\end{tabular} & 6.63 & 7.10 & 6.87 \\
\hline $\mathrm{T}_{6}$ & \multicolumn{3}{|c|}{\begin{tabular}{|l|l|l|}
7.347 .08 & 6.43 & 6.95 \\
\end{tabular}} & 6.97 & 6.95 & 6.96 & \multicolumn{2}{|c|}{\begin{tabular}{|l|l|}
7.447 .096 .51 \\
\end{tabular}} & \begin{tabular}{|l|l|}
6.51 & 7.01 \\
\end{tabular} & 6.97 & 7.01 & 6.99 \\
\hline $\mathrm{T}_{7}$ & \multicolumn{3}{|c|}{\begin{tabular}{|l|l|l|}
6.526 .33 & 6.26 & 6.37 \\
\end{tabular}} & 6.28 & 6.37 & 6.33 & \multirow{2}{*}{\multicolumn{3}{|c|}{\begin{tabular}{|l|l|l|}
6.586 .42 & 6.32 \\
7.306 .89 & 6.64 \\
\end{tabular}}} & 6.28 & 6.44 & 6.36 \\
\hline Mean & \multicolumn{3}{|c|}{\begin{tabular}{|l|l|l|}
7.126 .79 & 6.54 \\
\end{tabular}} & 6.54 & 6.82 & & & & & 6.54 & 6.94 & \\
\hline \multirow{3}{*}{$\mathrm{CD}_{(0.05)}$} & 7 & $T$ & 0.12 & $\mathrm{~T}$ & \multicolumn{2}{|l|}{0.34} & $\mathrm{~T}$ & $\Gamma$ & 0.34 & $\mathrm{~T}$ & \multicolumn{2}{|c|}{0.33} \\
\hline & I & D & 0.28 & $\mathrm{D}$ & \multicolumn{2}{|l|}{0.18} & \multicolumn{2}{|c|}{$\mathrm{D}$} & 0.22 & $\mathrm{D}$ & \multicolumn{2}{|c|}{0.18} \\
\hline & $\mathrm{T}\rangle$ & $x \mathrm{D}$ & NS & $\mathrm{T} \times \mathrm{D}$ & \multicolumn{2}{|l|}{ NS } & \multicolumn{2}{|c|}{$\mathrm{T} \times \mathrm{D}$} & NS & $\mathrm{T} \times \mathrm{D}$ & \multicolumn{2}{|l|}{ NS } \\
\hline
\end{tabular}

$* \mathrm{~T}=$ Tree; $\mathrm{D}=$ distance of application from tree trunk; $\mathrm{T} \times \mathrm{D}=$ interaction between tree and distance of application from tree trunk.

Table 5: Effect of organic manure and distance from tree trunk on soil organic carbon (\%) under different agroforestry system.

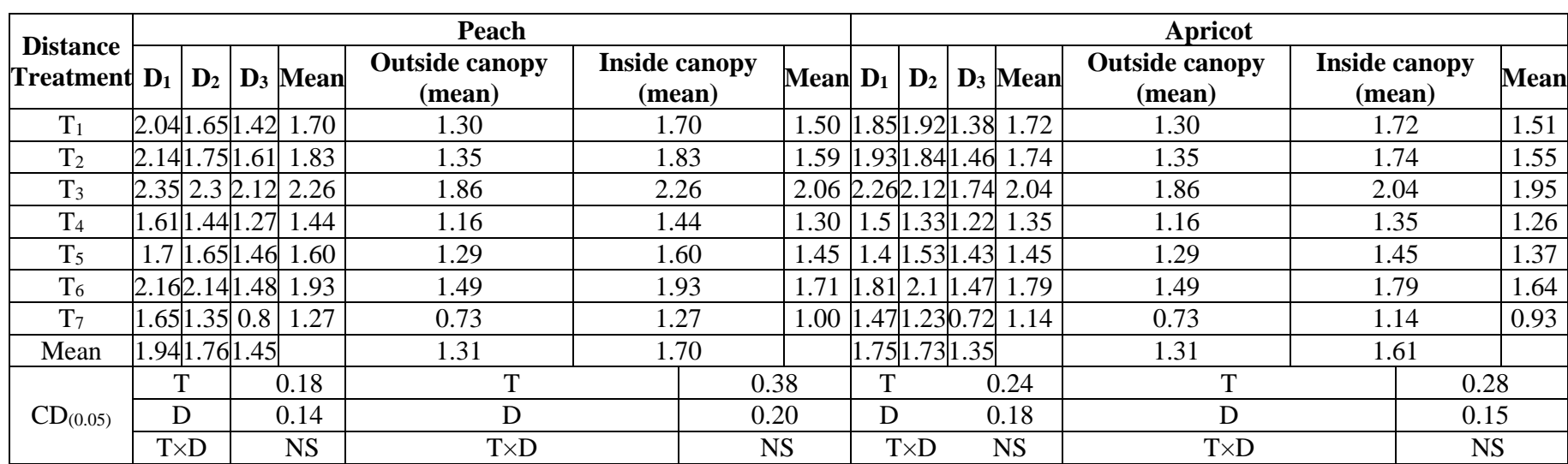

*T= Tree; $\mathrm{D}=$ distance of application from tree trunk; $\mathrm{T} \times \mathrm{D}=$ interaction between tree and distance of application from tree trunk

Table 6: Effect of organic manure and distance from tree trunk on available $\mathrm{N}(\mathrm{Kg} / \mathrm{ha})$ under different agroforestry system.

\begin{tabular}{|c|c|c|c|c|c|c|c|c|c|c|c|c|c|c|}
\hline \multirow{2}{*}{$\begin{array}{c}\text { Distance } \\
\text { Treatment }\end{array}$} & \multicolumn{7}{|c|}{ Peach } & \multicolumn{7}{|c|}{ Apricot } \\
\hline & $\mathbf{D}_{1}$ & $\mathbf{D}_{2}$ & D3 & Mean & $\begin{array}{c}\text { Outside } \\
\text { canopy (mean) }\end{array}$ & $\begin{array}{c}\text { Inside canopy } \\
\text { (mean) }\end{array}$ & Mean & $\mathbf{D}_{1}$ & $\mathbf{D}_{2}$ & $\mathbf{D}_{3}$ & Mean & $\begin{array}{c}\text { Outside } \\
\text { canopy (mean) }\end{array}$ & $\begin{array}{c}\text { Inside canopy } \\
\text { (mean) }\end{array}$ & Mean \\
\hline $\mathrm{T}_{1}$ & \multicolumn{4}{|c|}{\begin{tabular}{|l|l|l|l|}
318.85 & 313.04306 .69 & 312.86 \\
\end{tabular}} & 303.15 & 312.86 & 308.01 & \multicolumn{4}{|c|}{318.42322 .22307 .19315 .94} & 303.15 & 315.94 & 309.55 \\
\hline $\mathrm{T}_{2}$ & \multicolumn{4}{|c|}{343.61339 .80328 .34337 .25} & 312.13 & 337.25 & 324.69 & \multicolumn{4}{|c|}{\begin{tabular}{|l|l|l|}
346.78353 .28 & 316.65 & 338.91 \\
\end{tabular}} & 312.13 & 338.90 & 325.52 \\
\hline $\mathrm{T}_{3}$ & \multicolumn{4}{|c|}{354.97348 .09335 .48346 .18} & 305.99 & 346.18 & 326.08 & \multicolumn{4}{|c|}{355.42356 .47329 .40347 .10} & 305.99 & 7.10 & 326.5 \\
\hline $\mathrm{T}_{4}$ & \multicolumn{4}{|c|}{\begin{tabular}{|c|c|}
312.15 & 301.86299 .08 \\
304.36
\end{tabular}} & 300.00 & 304.36 & 302.18 & \multicolumn{4}{|c|}{8317.19205 .18299 .74274 .04} & 300.00 & 44 & 287.02 \\
\hline $\mathrm{T}_{5}$ & \multicolumn{4}{|c|}{321.61317 .50314 .37317 .83} & 306.50 & 317.83 & 312.16 & \multicolumn{4}{|c|}{$6313.89325 .54306,48315.30$} & 306.50 & 315.30 & 310.90 \\
\hline $\mathrm{T}_{6}$ & \multicolumn{4}{|c|}{\begin{tabular}{|c|c|c|}
337.42 & 331.03324 .18330 .88 \\
\end{tabular}} & 311.07 & 330.88 & 320.98 & \multicolumn{4}{|c|}{8335.82342 .65316 .95331 .81} & 311.07 & 331.81 & 321.44 \\
\hline $\mathrm{T}_{7}$ & \multicolumn{4}{|c|}{\begin{tabular}{|l|l|l|l|}
287.64 & 251.72 & 235.73 & 258.36 \\
\end{tabular}} & 270.40 & 258.36 & 264.38 & \multicolumn{4}{|c|}{8230.26299 .54266 .74265 .51} & 270.40 & 265.51 & 267.96 \\
\hline Mean & \multicolumn{4}{|c|}{325.18314 .72306 .27} & 301.32 & 315.39 & & \multicolumn{4}{|c|}{316.83314 .98306 .17} & 301.32 & 312.66 & \\
\hline \multirow{3}{*}{$\mathrm{CD}_{(0.05)}$} & \multicolumn{2}{|c|}{$\mathrm{T}$} & \multicolumn{2}{|c|}{21.66} & $\mathrm{~T}$ & \multicolumn{2}{|l|}{22.37} & \multicolumn{2}{|l|}{$\mathrm{T}$} & \multicolumn{2}{|c|}{38.39} & $\mathrm{~T}$ & \multicolumn{2}{|l|}{32.73} \\
\hline & \multirow{2}{*}{\multicolumn{2}{|c|}{$\begin{array}{c}\mathrm{D} \\
\mathrm{T} \times \mathrm{D}\end{array}$}} & \multicolumn{2}{|c|}{14.18} & $\mathrm{D}$ & \multicolumn{2}{|l|}{11.96} & \multicolumn{2}{|l|}{$\mathrm{D}$} & \multicolumn{2}{|c|}{ NS } & $\mathrm{D}$ & \multicolumn{2}{|l|}{ NS } \\
\hline & & $\mathrm{T} \times \mathrm{D}$ & \multicolumn{2}{|c|}{ NS } & $\mathrm{T} \times \mathrm{D}$ & \multicolumn{2}{|l|}{ NS } & $\mathrm{T} \times \mathrm{L}$ & & $\mathrm{N}$ & $\mathrm{S}$ & $\mathrm{T} \times \mathrm{D}$ & NS & \\
\hline
\end{tabular}

$* \mathrm{~T}=$ Tree; $\mathrm{D}=$ distance of application from tree trunk; $\mathrm{TxD}=$ interaction between tree and distance of application from tree trunk 
Table 7: Effect of organic manure and distance from tree trunk on available $\mathrm{P}(\mathrm{Kg} / \mathrm{ha})$ under different agroforestry system.

\begin{tabular}{|c|c|c|c|c|c|c|c|c|c|c|c|c|c|c|}
\hline \multirow[b]{2}{*}{$\begin{array}{c}\text { Distance } \\
\text { Treatment }\end{array}$} & \multicolumn{7}{|c|}{ Peach } & \multicolumn{7}{|c|}{ Apricot } \\
\hline & $D_{1}$ & $\mathbf{D}_{2}$ & $\mathbf{D}_{3}$ & Mean & $\begin{array}{c}\text { Outside canopy } \\
\text { (mean) }\end{array}$ & $\begin{array}{c}\text { Inside canopy } \\
\text { (mean) }\end{array}$ & Mean & $\mathbf{D}_{1}$ & $\mathbf{D}_{2}$ & $\mathbf{D}_{3}$ & Mean & $\begin{array}{c}\text { Outside canopy } \\
\text { (mean) }\end{array}$ & $\begin{array}{c}\text { Inside canopy } \\
\text { (mean) }\end{array}$ & Mean \\
\hline $\mathrm{T}_{1}$ & \multicolumn{4}{|c|}{49.7742 .4442 .4544 .89} & 45.26 & 44.89 & 45.08 & \multicolumn{4}{|c|}{\begin{tabular}{|l|l|l|l|}
49.08 & 42.33 & 44.89 & 45.43 \\
\end{tabular}} & 45.26 & 45.43 & 45.35 \\
\hline $\mathrm{T}_{2}$ & \multicolumn{4}{|c|}{\begin{tabular}{|l|l|l|l|l|}
52.48 & 45.4844 .52 & 47.49 \\
\end{tabular}} & 47.00 & 47.49 & 47.25 & 51.19 & 46.52 & 44.95 & 47.55 & 47.00 & 47.55 & 47.28 \\
\hline $\mathrm{T}_{3}$ & \multicolumn{4}{|c|}{\begin{tabular}{|l|l|l|}
55.0847 .2945 .13 & 49.17 \\
\end{tabular}} & 48.30 & 49.17 & 48.73 & 53.88 & 46.52 & 43.86 & 48.09 & 48.30 & 48.09 & 48.19 \\
\hline $\mathrm{T}_{4}$ & \multicolumn{4}{|c|}{\begin{tabular}{|l|l|l|l|}
48.7838 .94 & 38.81 & 42.18 \\
\end{tabular}} & 42.28 & 42.18 & 42.23 & 47.56 & 38.22 & 40.08 & 41.95 & 42.28 & 41.95 & 42.12 \\
\hline $\mathrm{T}_{5}$ & \multicolumn{4}{|c|}{\begin{tabular}{|l|l|l|}
50.9243 .4642 .62 & 45.67 \\
\end{tabular}} & 43.81 & 45.67 & 44.74 & 51.34 & 40.19 & 41.18 & 44.24 & 43.81 & 44.24 & 44.02 \\
\hline $\mathrm{T}_{6}$ & \multicolumn{4}{|c|}{53.3644 .3143 .4547 .04} & 43.86 & 47.04 & 45.45 & 53.25 & 40.11 & 41.96 & 45.11 & 43.86 & 45.11 & 44.48 \\
\hline $\mathrm{T}_{7}$ & \multicolumn{4}{|c|}{\begin{tabular}{|l|l|l|l|l|}
40.7429 .05 & 29.43 & 33.07 \\
\end{tabular}} & 34.49 & 33.07 & 33.78 & 39.56 & 24.90 & 30.04 & 31.50 & 34.49 & 31.50 & 32.99 \\
\hline Mean & \multicolumn{4}{|c|}{50.1641 .5740 .92} & 43.57 & 44.22 & & 49.41 & 39.83 & 40.99 & & 43.57 & 43.41 & \\
\hline \multirow{3}{*}{$\mathrm{CD}_{(0.05)}$} & & $\Gamma$ & & 2.77 & $\mathrm{~T}$ & \multicolumn{2}{|l|}{2.47} & 7 & $\Gamma$ & & 1.38 & $\mathrm{~T}$ & \multicolumn{2}{|l|}{2.70} \\
\hline & & 5 & & 1.81 & $\mathrm{D}$ & \multicolumn{2}{|l|}{ NS } & \multicolumn{2}{|c|}{$\mathrm{D}$} & & .90 & $\mathrm{D}$ & \multicolumn{2}{|l|}{ NS } \\
\hline & \multicolumn{2}{|c|}{$\mathrm{T} \times \mathrm{D}$} & & NS & $T \times D$ & \multicolumn{2}{|c|}{ NS } & \multicolumn{2}{|c|}{$\mathrm{T} \times \mathrm{D}$} & & 2.39 & $\mathrm{~T} \times \mathrm{D}$ & \multicolumn{2}{|l|}{$\mathrm{NS}$} \\
\hline
\end{tabular}

$* \mathrm{~T}=$ Tree; $\mathrm{D}=$ distance of application from tree trunk; $\mathrm{TxD}=$ interaction between tree and distance of application from tree trunk.

Table 8: Effect of organic manure and distance from tree trunk on available $\mathrm{K}(\mathrm{Kg} / \mathrm{ha})$ under different agroforestry system.

\begin{tabular}{|c|c|c|c|c|c|c|c|c|c|c|c|c|c|c|}
\hline \multirow{2}{*}{$\begin{array}{c}\text { Distance } \\
\text { Treatment }\end{array}$} & \multicolumn{7}{|c|}{ Peach } & \multicolumn{7}{|c|}{ Apricot } \\
\hline & $D_{1}$ & $\mathbf{D}_{2}$ & $\mathbf{D}_{3}$ & Mean & $\begin{array}{c}\text { Outside } \\
\text { canopy (mean) }\end{array}$ & $\underset{(\text { mean })}{\text { Inside canopy }}$ & Mean & $\mathbf{D}_{1}$ & $\mathbf{D}_{2}$ & $\mathbf{D}_{3}$ & Mean & $\begin{array}{c}\text { Outside } \\
\text { canopy (mean) }\end{array}$ & $\begin{array}{c}\text { Inside canopy } \\
\text { (mean) }\end{array}$ & Mean \\
\hline $\mathrm{T}_{1}$ & \multicolumn{4}{|c|}{\begin{tabular}{|l|l|l|}
286.63 & 278.17 & 269.08 \\
277.96 \\
\end{tabular}} & 230.52 & 277.96 & \multicolumn{5}{|c|}{ 254.24|250.96|241.26231.56|241.26 } & 230.52 & 241.26 & 235. \\
\hline $\mathrm{T}_{2}$ & \multicolumn{4}{|c|}{\begin{tabular}{|l|l|l|}
327.04 & 300.45287 .19 & 304.89 \\
\end{tabular}} & 222.76 & 304.89 & \multicolumn{5}{|c|}{\begin{tabular}{|l|l|l|l|l|}
263.83 & 257.32 & 249.03 & 239.44 & 248.60 \\
\end{tabular}} & 222.76 & 248.60 & 235. \\
\hline $\mathrm{T}_{3}$ & \multicolumn{4}{|c|}{\begin{tabular}{|l|l|l|l|}
356.40 & 330.92 & 305.78 & 331.03 \\
\end{tabular}} & 257.83 & 331.03 & 294.43 & \multicolumn{4}{|c|}{\begin{tabular}{|l|l|}
278.96261 .42255 .86 & 265.41
\end{tabular}} & 257.83 & .41 & 261.6 \\
\hline $\mathrm{T}_{4}$ & \multicolumn{4}{|c|}{$265.84|265.17244 .74| 258.58$} & 227.58 & 258.58 & \multirow{3}{*}{\begin{tabular}{|l|}
243.08 \\
245.65 \\
259.45
\end{tabular}} & \multicolumn{4}{|c|}{232.01230 .37229 .13230 .50} & 227.58 & 230.50 & 229.0 \\
\hline $\mathrm{T}_{5}$ & \multicolumn{4}{|c|}{ 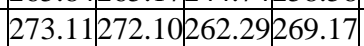 } & 222.14 & 269.17 & & \multicolumn{4}{|c|}{ 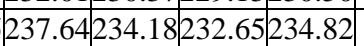 } & 222.14 & 234.82 & 228.4 \\
\hline $\mathrm{T}_{6}$ & \multicolumn{4}{|c|}{$279.63|309.68269 .75| 286.35$} & 232.54 & 286.35 & & 248.09 & 237.6 & 235.87 & 7240.54 & 232.54 & 240.54 & 236. \\
\hline $\mathrm{T}_{7}$ & \multicolumn{4}{|c|}{\begin{tabular}{|l|l|l|}
249.40 & 219.04219 .16 & 229.20 \\
\end{tabular}} & 201.79 & 229.20 & 215.50 & \multirow{2}{*}{\multicolumn{4}{|c|}{\begin{tabular}{|l|l|l|l|l|}
228.74 & 222.21 & 221.25 & 224.07 \\
247 & 67 & 239 & 45 & 235 \\
\end{tabular}}} & 201.79 & 224.07 & 212. \\
\hline Mean & \multicolumn{4}{|c|}{291.15282 .22265 .43} & 227.88 & 279.60 & & & & & & 227.88 & 240.74 & \\
\hline \multirow{3}{*}{$\mathrm{CD}_{(0.05)}$} & \multicolumn{2}{|c|}{$\mathrm{T}$} & \multicolumn{2}{|c|}{29.76} & $\mathrm{~T}$ & \multicolumn{2}{|l|}{29.28} & & T & & 7.50 & $\mathrm{~T}$ & \multicolumn{2}{|l|}{26.32} \\
\hline & \multicolumn{2}{|c|}{ D } & \multicolumn{2}{|c|}{19.48} & $\mathrm{D}$ & \multicolumn{2}{|l|}{15.65} & & 5 & \multicolumn{2}{|c|}{ NS } & $\mathrm{D}$ & \multicolumn{2}{|l|}{ NS } \\
\hline & \multicolumn{2}{|c|}{$\mathrm{T} \times \mathrm{D}$} & \multicolumn{2}{|c|}{ NS } & $\mathrm{T} \times \mathrm{D}$ & \multicolumn{2}{|l|}{ NS } & & $x \mathrm{D}$ & & NS & $T \times D$ & NS & \\
\hline
\end{tabular}

$* \mathrm{~T}=$ Tree; $\mathrm{D}=$ distance of application from tree trunk; $\mathrm{TxD}=$ interaction between tree and distance of application from tree trunk.

\section{References}

1. Karlen DL, Mausbach, MJ, Doran JW, Cline RG, Harris RF, and Schuman GE. Soil quality: A concept, definition, and framework for evaluation. Soil Science Society of America Journal 1997;61:4-10.

2. Oliver K, Njira W, Nabwami J. Soil management practices that improve soil health: Elucidating their implications on biological indicators. Journal of Animal and Plant Sciences 2013;18(2):2750-2760.

3. Semwal DP, Uniyal PL, Bahuguna YM, Bhatt AB. Soil nutrient storage under different forest types in a part of Central Himalayas, India. Annals of Forestry 2009;17(1):43-52.

4. Araujo ASF, Leite LFC, Iwata BF, Jr M de AL, Xavier GR, Figueiredo MVB. Microbiological process in Agroforestry systems: A review. Agronomy for Sustainable Development 2012;32:215-226.

5. Sarvade S, Singh R, Vikas G, Kachawaya DS, Khachi B. Agroforestry: an approach for food security. Indian Journal of Ecology 2014;41(1):95-98.

6. FAO. Healthy soils are the basis for healthy food production. Rome, Italy 2015,4.

7. Nair PKR. Role of trees in soil productivity and conservation. Soil productivity aspects of agro-forestry. The International Council for Research in Agro-Forestry. Nairobi 1984,85.

8. Dutt V and Thakur PS. Bioeconomics of cropping systems combining medicinal and aromatic herbs with commercial timber tree species. The Indian Journal of Agroforestry 2004;6:1-7.

9. Albiach R, Canet R, Pomares F, Ingelmo F. Microbial biomass content and enzymatic activities after the application of organic amendments to a horticulture soil. Bioresources Technology 2000;75:43-48.

10. Raina NS, Rafiq M, Sood KK, Bali AS, Gupta SK, Sehga S. Growth and yield of Ocimum sanctum in response to integrated nutrient management and plant spacing. Indian Journal of Agronomy 2013; 58(1):129-132.

11. Lucchesi ME, Chemat F, Smadja J. Solvent-free microwave extraction of essential oil from aromatic herbs: Comparison with conventional hydro-distillation. Journal of Chromatography A 2004;1043(2):323-32.

12. Palekar S. Text book on Shoonya Bandovalada naisargika Krushi, published by Swamy Anand, Agri Prakashana, Bangalore,2006.

13. Basavaraj K, Devakumar N, Sheshadri T. Influence of farm yard manure, jeevamrutha and panchagavya on growth and yield of french bean (Phaseolus vulgaris L.). Mysore Journal of Agricultural Sciences 2006;50:279283.

14. Jackson ML. Soil chemical analysis. Prentice Hall of Indian Pvt. Ltd., New Delhi 1973,498.

15. Walky A, Black TA. An estimation method for determining soil organic matter and proposed modification of the chromic acid titration method. Soil Science 1934;37:250-260.

16. Subbiah BV, Asija GL. Rapid method for estimation of available nitrogen in soils. Current Science 1956;25:259260.

17. Olsen R, Cole CV, Wantanable FS, Dean LA. Estimation of available $\mathrm{P}$ in soil by extraction with sodium bicarbonate. Washington, US Deptt. of Agricultural 1954;939:19. 
18. Merwin HD, Peech M. Exchangeable of soil potassium in the sand, silt and clay fractions, as influenced by the nature of the complementary exchangeable cations. Soil Science American Proceedings 1951;15:125-128.

19. Gomez LA, Gomez AA. Statistical Procedure for Agricultural Research. John Wiley and Sons, Singapore 1984,680.

20. Tripathi P. Effect of organic manures on yield and biomass production of medicinal and aromatic plants under peach based agroforestry system. M.Sc. Thesis, UHF, Solan, HP 2012.

21. Acharya CL, Bishnoi SK, Yadhuvanshi HS. Effect of long term application of fertilizers and organic and inorganic amendments under continuous cropping on soil physical and chemical properties in Alfisols. Indian Journal Agriculture Science 1988;58:509-516.

22. Yogeshwari. Crop production and physico-chemical characteristics of soils under Toona ciliata M. Roem trees in mid hill conditions of Himachal Pradesh. M.Sc. Thesis. UHF, Nauni, Solan 2015.

23. Bowen WT, Quitena JQ, Pereira J, Bouldin DR, Reid WS, Lathwell DJ. Screening green manures as nitrogen sources to successing non-legume crops. Plant Soil 1988;111:75-89.

24. Khybri ML, Gupta RK, Ram S, Tomar HPS. Crop yields of rice and wheat grown in rotation as intercrops with 3 tree species in the outer hills of Western Himalaya. Agroforestry Systems 1992;17:193-204.

25. Bhat SA. Effect of tree spacing and organic manures on growth and yield of vegetable crops under Melia composita willd based agroforestry system. Ph.D Thesis. UHF, Nauni, Solan 2015.

26. Bellakki MA, Badanur VP. Long-term effect of integrated nutrient management on properties of Vertisol under dryland agriculture. J Indian Soc. Soil Sci 2015;45:438-442.

27. Balaji SK. Effect of vermicompost on growth and flower yield of China aster (Callistephus chinensis). M. Sc. (Agri.) Thesis. Univ. Agric. Sci. Dharwad (India) 1994.

28. Chandrakala M. Effect of fym and fermented liquid manures on yield and quality of chilli (Capsicum annuum L.). M.Sc. Thesis, Department of Soil science and agricultural chemistry, University of Agricultural Sciences, Dharwad 2008.

29. Gulabrao YA. Carbon sequestration and effect of organic manures on yield and biomass production of medicinal and aromatic plants under kinnow based agroforestry system. M.Sc. Thesis. UHF, Solan, HP 2016.

30. Sharma SK, Sharma SN. Integrated nutrient management for sustainability of rice (Oryza sativa)-wheat (Triticum aestivum) cropping system. Indian Journal of Agriculture Science 2002;72:573-576. 